\title{
Un EDIFICIO DE USO ARTESANAl EN El POBlado IBÉRICO DEL PiCO DE LOS Ajos (YÁtova, València)
}

DAVID QUIXAL SANTOS ${ }^{1} \oplus$, CONSUELO MATA PARREÑO ${ }^{2} @$ ANA SERRANO CASTELLANO $^{1}$

(1) Dpt. Prehistòria, Arqueologia i $\mathrm{H}^{\mathrm{a}}$ Antiga. Universitat de València.david.quixal@uv.es, asecas@alumni.uv.es

(2) GRAM. Dpt. Prehistòria, Arqueologia i H ${ }^{\mathrm{a}}$ Antiga. Universitat de València. consuelo.mata@uv.es

En trabajos precedentes se expusieron las características generales de este poblado fortificado ubicado en la cima de Sierra Martés, declarado BIC y conocido por su colección de plomos escritos en signario ibérico (Fletcher 1980; Díes y Gimeno 1995; Quixal 2010). En 2017 se acometió la primera campaña de excavación, tras años y años de expolio incontrolado, financiada por el Ayuntamiento de Yátova (Quixal et al. 2018). La misma se centró en el Sector 1, uno de los más afectados por la actividad clandestina, que es la primera plataforma del yacimiento comenzando por el NO. Se realizaron diversos sondeos que permitieron documentar dos fases de ocupación: una en los ss. VII-VI, posible momento fundacional, y otra en los ss. III-I a.C., fase de máxima expansión del mismo. En este sector destacó la presencia de un largo muro longitudinal, de unos $30 \mathrm{~m}$ de longitud, que atravesaba la plataforma más o menos por el centro, definiendo hacia el $\mathrm{N}$ toda una serie de espacios o departamentos. Se excavó parcialmente uno de ellos, por lo que en 2018 se decidió realizar un par de sondeos paralelos para comprobar el estado de conservación de los siguientes. Al tratarse de un sector todavía en proceso de excavación y estudio, remitimos a futuras publicaciones.
No obstante, el grueso de los trabajos de $2018^{1}$ se desarrolló en un área diferente del yacimiento, que es la que vamos a presentar en estas líneas: el Sector 4. Un espectacular espolón o balcón natural hacia el S, hacia el llano de Venta Gaeta, justo en la zona de acantilados más abrupta (fig. 1). El pequeño sondeo realizado allí en 2017 animó a continuar en 2018 la excavación de la práctica totalidad de dicho espolón, al tratarse de un área no afectada por acciones clandestinas. En la misma hallamos un edificio con cuatro espacios (E1, E2, E3, E4) delimitados por una serie de muros con orientación NO-SE y NE-SO (fig. 2). La estructura principal es un muro compuesto por grandes losas verticales (4005) y su continuación hacia el SE (4008). La alineación es completa y seguramente se trate del eje principal. Del mismo surgen en perpendicular dos muros (4006 y 4016) que, aunque parecen conformar una cruz perfecta, no es así, ya que la alineación en este caso no es total, quedando dos L invertidas. Ello nos hace dudar de si todas las construcciones pertenecen al mismo momento o se trata de diferentes fases constructivas, debido a la fuerte erosión del lugar.

De los cuatro espacios configurados, uno claramente estaría cubierto, delimitado por los muros 4006, 4008, 


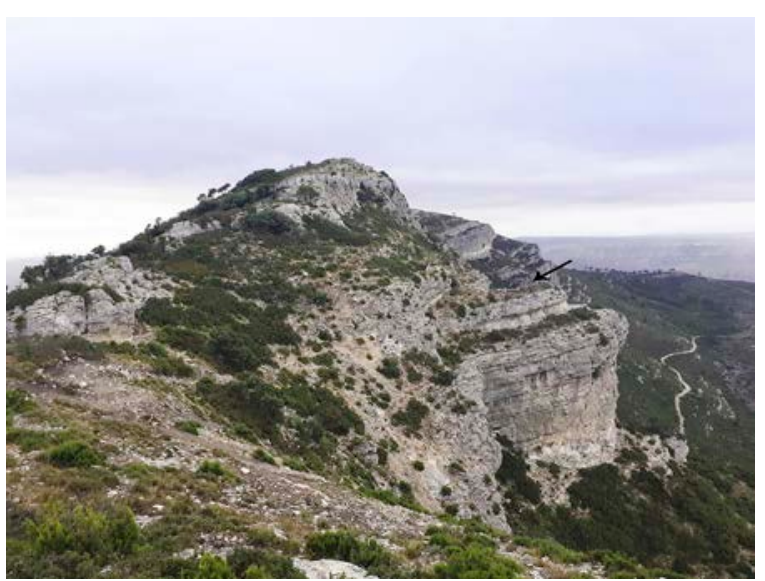

Fig. 1: Vista del espolón en el Sector 4 del Pico de los Ajos.

4018 y 4019 . Éstos se apoyan directamente sobre la roca y en ocasiones, como es el caso del 4019, se sirven de un recorte en la misma. En el ángulo septentrional encontramos uno de estos recortes justo en la esquina, no sabemos si a modo de escalón o de pequeño banco. El grosor de los muros es considerable, teniendo en ocasiones doble paramento y utilizando mampuestos de gran tamaño, algunos de ellos escuadrados. Su estado de conservación impide conocer con seguridad por dónde se realizaría el acceso, dada la complejidad orográfica del espolón. Podría ser por el NE, desde una cota más alta, o por el SE, siguiendo alguna cota de nivel inferior. Seguramente se trate de esto último, aprovechando la zona en la que el muro 4018 carece de continuidad y encontramos directamente la roca natural haciendo de escalón. Además, el muro 4008 continúa hacia el SE, ladera abajo, quizás conformando algún tipo de entrada. La estratigrafía de esta estancia es muy simple dada la escasa potencia conservada: bajo del nivel superficial encontramos directamente el nivel de abandono. La roca, acondicionada y posiblemente con algún tipo de preparación, actuaba como pavimento. En su interior hemos encontrado abundante material cerámico y metálico, como luego veremos.

La E1 no podemos considerar que fuera un espacio cerrado, sino probablemente un área semicubierta, completada con pequeños muretes. Del mismo modo, en este lugar se ha documentado un agujero de poste que serviría como solución de urgencia para reforzar alguno de los muros. La estratigrafía es calcada al caso anterior, con un nivel superficial cubriendo directamente el nivel de abandono y la roca actuando de pavimento.

Los dos espacios ubicados al S del muro 4005-4008 presentan marcadas similitudes y problemáticas. Para empezar, fue imposible excavarlos de forma completa hasta el vértice del espolón por motivos de seguridad. Del mismo modo, es la zona más expuesta a la erosión, por lo que su conservación es peor. La E3 probablemente

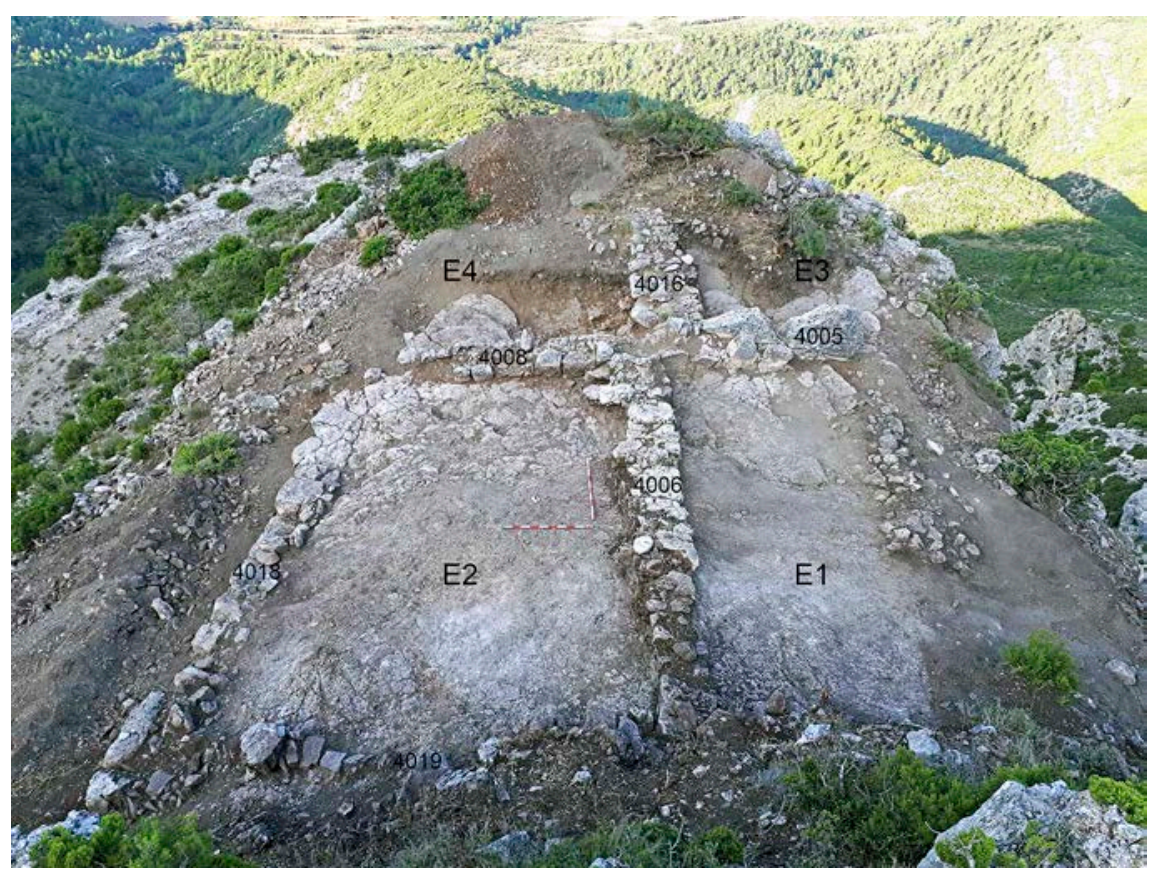

Fig. 2: Excavación en el Sector 4. 
sería un espacio cubierto o semicubierto, mientras que la E4 es complicada de interpretar si no se puede terminar la excavación del conjunto. Apenas se ha podido recuperar el nivel de abandono y el grueso del material aparece en el estrato superficial. Los niveles conservados servirían de relleno para acondicionar las irregularidades de la roca natural. Probablemente sean anteriores a la construcción de todo el edificio, de ahí el interés del estudio de sus materiales y su datación. El conjunto de cerámicas es reducido, por desgracia, si bien se han podido recuperar abundantes carbones.

En resumen, tenemos un gran departamento (E2) que constituiría la construcción principal del sector, completado con una serie de espacios abiertos o semicubiertos con funcionalidad auxiliar. Las estructuras ocupan la práctica totalidad del espolón, si bien los desprendimientos producidos en el transcurso de los siglos han alterado su fisonomía original. El acceso, complejo por las características orográficas, posiblemente se realizase por el SE. No obstante, la erosión sufrida por el lugar, la escasa potencia conservada y la imposibilidad de excavar por completo la totalidad del terreno impiden una interpretación más precisa de este peculiar edificio.

Derivado de todos estos trabajos en el Sector 4, hemos obtenido un destacable volumen de materiales arqueológicos, principalmente cerámicos ${ }^{2}$. La cerámica presenta un alto grado de fragmentación y desgaste, posiblemente por el propio carácter expuesto del espolón.
Siguiendo la tipología sobre cerámica ibérica de Mata y Bonet (1992), abundan los recipientes de los Grupos I y II (almacenaje y tareas domésticas), mientras que llama la atención la escasez de piezas de vajilla de mesa, cerámica de cocina u otros elementos auxiliares, grupos que podrían relacionarse con la existencia de una unidad doméstica. De igual forma, se han podido recuperar numerosos fragmentos de ánfora itálica republicana procedente de la Campania. Sin embargo, el material más destacado es un conjunto de elementos metálicos de hierro, bronce y plomo que permite aproximarse a la funcionalidad del edificio (fig. 3). Se han podido documentar dos posibles protolingotes de hierro con forma curvada (fig. 3, 1), tres ponderales (fig. 3, 2), tres espátulas lanceoladas con el vástago doblado (fig. 3, 3), diversas fíbulas completas e incompletas (fig. 3, 4), una hoja de tijeras (fig. 3, 5), un semi ibérico de Kese (fig. 3, 6) y numerosos elementos de carpintería. Otras piezas destacadas son un pequeño fragmento de plomo doblado y en el que se aprecian caracteres ibéricos por ambas caras (Pico de los Ajos VII); así como una cuenta de pasta vítrea. Apenas se han conservado restos de fauna, pero se han recogido muestras de sedimento para estudios paleobotánicos.

A la hora de interpretar la funcionalidad de este edificio del Sector 4, diversos son los factores que debemos tener en cuenta. En primer lugar, el propio carácter escarpado del emplazamiento, que lo convertiría en un lugar
Fig. 3: Objetos metálicos hallados en el transcurso de las excavaciones.

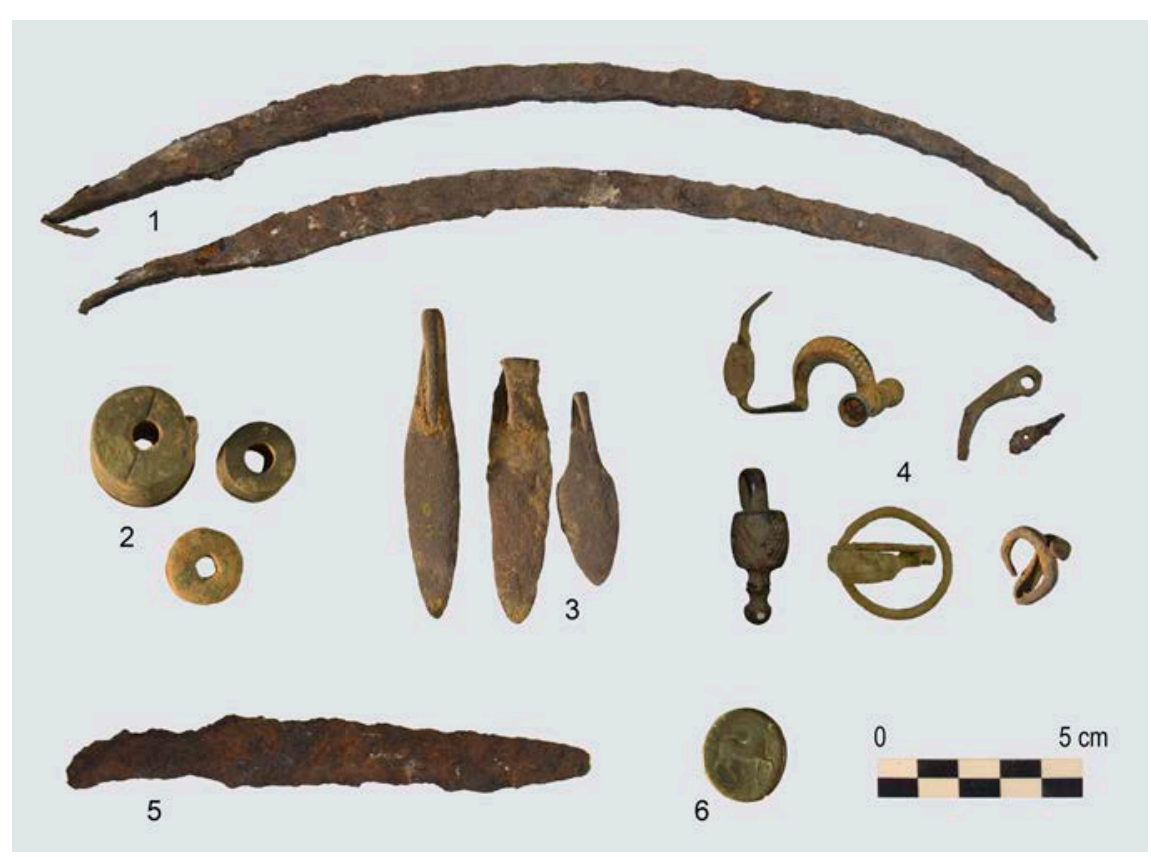


incómodo para vivir. En segundo lugar, el conjunto de materiales cerámicos, con abundantes recipientes para almacenaje, pero pocos indicios de uso doméstico (cocina, vajilla, elementos de telar, etc.). En tercer lugar, la fisonomía de los espacios documentados, con un departamento principal, cubierto, donde se concentra el material, alternando con posibles áreas abiertas o semicubiertas. Y, por último, el interesante lote de objetos metálicos, principalmente de bronce, muchos de ellos doblados, rotos o incompletos. Piezas todas ellas amortizadas y recuperadas para su refundición.

Por todo ello, planteamos que estamos delante de un depósito metalúrgico para su almacenaje y refundición, localizado en un área artesanal apartada de la zona de hábitat. Este tipo de áreas auxiliares, separadas del hábitat y dedicadas al almacenaje o a la metalurgia las hemos documentado también en otros asentamientos de cronología semejante (Quixal et al. 2010). Del mismo modo, actividades de reciclaje y refundición de metales están bien documentadas en otros ámbitos mediterráneos antiguos (Delfino 2014; Figueiredo et al. 2010). Los materiales, tanto por las formas ibéricas tardías, las importaciones de ámbito itálico y los elementos metálicos, nos permiten datarlo bastante bien en los ss. II-I a.C.

\section{NOTAS}

1. La campaña se desarrolló del 1 al 17 de agosto del 2018 por parte de un equipo compuesto por los codirectores de la excavación, C. Mata y D. Quixal, juntamente con un nutrido grupo de graduados y estudiantes de Historia de la Universitat de València: A. Serrano, J. Alberola, J. L. Azorín, S. Beneyto, R. Caballero, B. Castro, I. Civera, D. Corachán, M. Frías, I. García, C. Giménez, J. Gutiérrez, S. Hernández, E. López, C. Martínez, G. Martínez, S. Micó, D. Navarro, R. Pardo, D. Pérez, M. Sánchez, P. Valero y J. Vicent. De nuevo, los trabajos fueron sufragados íntegramente por el Ayuntamiento de Yátova.

2. Su estudio constituye el tema de Trabajo Final de Máster de A. Serrano.

\section{BIBLIOGRAFÍA}

DELFINO, D. (2014): Bronze recycling during the Bronze Age: some consideration about two metallurgical regions, $A n$ trope 1, Metodologias de Trabalho Arqueológico. Campo, Laboratório, Divulgação, 121-143.

DÍES, E.; GIMENO, L. (1995): El sistema defensivo de la zona SE del yacimiento ibérico del Pico de los Ajos (Yátova, Valencia), Sagvntvm-PLAV 29, 85-92.

FIGUEIREDO, E.; SILVA, R.; SENNA-MARTINEZ, J.; FÁTIMA, M.; BRAZ, F. M.; INÊS, J. L. (2010): Smelting and recycling evidences from the Late Bronze Age habitat site of Baiões (Viseu, Portugal), Journal or Archaeological Science 37, 1623-1634. DOI: https://doi.org/10.1016/j.jas.2010.01.023

FLETCHER, D. (1980): Los plomos ibéricos de Yátova, Serie Trabajos Varios. S.I.P. 81, València.

MATA, C.; BONET, H. (1992): La cerámica ibérica: ensayo de tipología, Estudios de arqueología ibérica y romana: homenaje a Enrique Pla Ballester, Serie Trabajos Varios. S.I.P. 89 , València, 117-174.

QUIXAL, D. (2010): El Pico de los Ajos (Yátova) y el poblamiento ibérico en torno a los ríos Magro y Mijares, Revista de Estudios Comarcales Buñol-Chiva 9, 25-35.

QUIXAL, D.; MATA, C.; ALBELDA, V.; PÉREZ REYES, A. (2010): Primera campaña de excavación en el asentamiento ibérico final de la Casa de la Cabeza (Requena, València), Sagvntvm-PLAV 42, 115-117.

QUIXAL, D.; MATA, C.; MARTÍNEZ, C. (2018): Primera campaña de excavación en el poblado ibérico del Pico de los Ajos (Yátova, Valencia), Sagvntvm-PLAV 50, 247-250. DOI: https://doi.org/10.7203/SAGVNTVM.50.12503 